\title{
Facts about probiotics
}

\begin{abstract}
Numerous claimed benefits of using probiotics invade the Media. However, Health Authorities in charge rejected all petitions of health claims due to lack of supportive research. Reaching a final decision about this issue seems not easy, with thousands of articles and researches about probiotics in medical literature present similar illusive view. In this paper, we try to illustrate some of the unbiased studies about probiotics possible benefits. It seems that each effect of probiotics is strain specific .so, generalization deepens that contradiction. Probiotic is considered a promising biologic medication that worth more research and strain -disease relationship understanding.
\end{abstract}

Volume 6 Issue 2 - 2017

\section{Gihad Al-Saeed, Tamer Rizk}

Department of Pediatrics and Pediatric Neurology,Al-Takhassusi Hospital, Dr. Sulaiman Al-Habib Medical Group, Saudi Arabia

Correspondence: Gehad Al-Saeed, Department of Pediatrics, Al-Takhassusi Hospital, Dr. Sulaiman Al-Habib Medical Group, Riyadh, Saudi Arabia, Email gehadalsaeed@yahoo.com

Received: January 27, 2016 | Published: February 07, 2017

\section{Introduction}

In 1907 Élie Metchnikoff; a Russian zoologist best known for his pioneering research in immunology; put the auto intoxication theory and suggested that changing the harmful intestinal flora with beneficial one can prevent aging. ${ }^{1}$

First commercially sold diary-based probiotic (Yakult) in 1935 was a mixture of fermented skimmed milk and Lactobacillus casei Shirota, first launched in japan. ${ }^{1}$

Today, we have more than 500 microorganisms isolated and used as probiotics in dairy products, breakfast cereal, snack bars, and many other food stuff and beverages.

Numerous claimed benefits of using probiotics, from relieving intestinal discomfort, to cancer prevention, immunodeficiency treatment and most if not all chronic diseases management.

The European Food Safety Authority has rejected all petitions of health claims on probiotics due to insufficient research, the American Food and Drug Association (FDA) sent warning letters to probiotics manufacturers for using exaggerated claims which are not supported by clinical evidence of efficacy. Despite that, Western Europe still considered the leading consumer of probiotics (8.3billion dollars in 2014 only).

Since 1980 great care about probiotics and its use in medicine started to grow with thousands of studies, most of them with inconclusive results, or not unbiased. In this paper, we try to draw attention to some possible indications of probiotics that have been supported by unbiased studies.

\section{Purposes of this paper}

1. Better understanding about probiotics.

2. Push for more trials about the mentioned aspects of use.

3. Describe the correct way of prescription.

4. Deliver this message scientifically and professionally to our patients.

\section{Definition of probiotics}

Probiotic is a live microorganism which confer a healthy benefit on the host when administered in adequate amounts. It can be bacteria, molds or yeast. ${ }^{2}$
Effects of probiotics are strain-specific and cannot be extended to other probiotics even if it belongs to the same group. ${ }^{3}$

\section{Mechanism of action of probiotics}

After oral consumption, it seems that each strain has one or more of the following effects, in a very specific way:

1. Direct antagonism to pathogens ${ }^{4}$

2. Barrier function and prevention of adhesion ${ }^{4}$

3. Competition for nutrients and prebiotics. ${ }^{5}$

4. Immunity stimulation or modulation: like increased mucosal immunoglobulin A (IGA), blood immunoglobulin G (IGG) and decrease immunoglobulin E (IGE). Increase T-Helper lymphocyte 1 (TH1) cytokines (Interferon (IFN), Tumor Necrotizing Factor (TNF), Interleukin (IL-6), and decrease T-Helper lymphocyte2 (TH2) cytokines (IL 4, IL5, IL3). ${ }^{5,6}$

5. May act as a facilitator modifying the activity of other gut bacteria like (lactobacillus Rhamnous GG). ${ }^{7-9}$

\section{Normal gastrointestinal (GI) microbiota}

At least 1150 different species of bacteria and molds form together the complex and metabolically active ecosystem known as the GI Microbiota. The composition of GI Microbiota is influenced by age, diet, and disease. In terms of ecological succession, the Bifidobacterium dominate microbiota of the infant changes over time into Bacteroides and Firmictures-dominate microbiota of the adult. After age 60years most strains of GI Microbiota are Bacteroides and clostridia cluster $5 .{ }^{10}$ Those who eat plenty of protein and animal fats typical of Western diet have predominantly Bacteroides bacteria (Enterotype1), while for those who consume more carbohydrates especially fibre, the Prevotella species dominate (Enterotype2). ${ }^{11}$ Perturbations in the gut microbial ecosystem have been associated with chronic GI diseases, DiabetesType 1 and2, obesity, and antibiotic use. (10)

\section{Probiotics in practice}

\section{Infant colic}

a. 4 trails about the rule of different types of probiotics confirmed decrease in crying time ranged between 30 to $55 \%$ when given during the first 3 months of life.

b. High doses not less than $109 \mathrm{CFU} /$ day always needed to get a symptomatic result for a period more than 6weeks. 
Improvement included: constipation, abdominal pain, and distension in $67 \%$ of the treated group. ${ }^{12}$

c. However, a study by Valerie Sung using Lactobacillus Reuteri DSM17938 on a community sample of breast fed and formula fed infants did not find any benefit. ${ }^{13}$

\section{Antibiotic associated diarrhea (AAD)}

a. AAD results from imbalance in the colonic microbiota caused by antibiotic therapy. Reduction in Bifidobacterium, Bacteroides, and Prevotella has been reported in addition to increase in clostridia species and Lactobacilli species.

b. Probiotics have a good prophylactic and therapeutic effect when given in a dose more than $5 \times 109 \mathrm{CFU} / \mathrm{DAY} .{ }^{9}$ Restoration of the microbiota and curing CDAD (clostridium difficile associated diarrhea) by fecal microbiota transplantation (FMT) in recent years presents a novel therapeutic strategy. ${ }^{10}$

c. Probiotics reduce the risk of necrotizing colitis and mortality in infants by $50 \% .^{14}$

\section{Gastroenteritis}

Probiotics can be used in prevention and therapy of childhood diarrhea. Effective probiotics should be resistant to gastric acid and bile salts, able to adhere to epithelia and has antimicrobial activity. Probiotics reduced the duration of diarrhea by 25 hours. It has a beneficial effect in Rota virus gastroenteritis.,

\section{Inflammatory bowel disease (IBD)}

In most cases of IBD an evidence of Dysbiosis of the GI microbiota has been described with less concentration of anti-inflammatory strains like Faecali bacterium Prausnitzii.

Extensive medical literature support the principal that modulating microbiota can suppress the pro inflammatory cytokines like (IFG, TNF, IL 12) in the intestine and induce secretion of IL10 and FXP3 in the blood in healthy volunteers. In one study using Bacteroides infantis 35624 strain, the IL10/IL12 Ratio normalized in treated patients.

Systematic review of controlled trails has suggested efficacy of probiotics used by rectal enema in maintaining remission in Ulcerative colitis. In contrast results were less impressive with little efficacy in Crohn disease (CD) ${ }^{15,16}$

\section{Food allergy}

Clostridium can enhance immune cells to produce IL22 which is known to decrease the intestinal lining permeability. Clostridia can minimize allergen exposure and prevent sensitization-a key step in the development of food allergy. this prophylactic and therapeutic effect on food allergy was supported by Food Allergy Research\& Education, and Chicago university. ${ }^{2,5}$

\section{Atopic dermatitis (AD) and asthma}

Staphylococcus aureus biofilms play an important role in AD Topical probiotic extract has been developed with very promising results, same efficacy like topical steroids, and better than topical anti staph antibiotics.

Administration of oral probiotics for high risk infants decreased significantly the incidence of $\mathrm{AD}$ during the first 2 years when compared with placebo group, although they were not found to change the inflammatory markers. ${ }^{6}$
Several in vitro studies suggest a role for probiotics in treating asthma and allergic rhinitis by modulating the immune system back to TH1. Till now no in vivo convincing study. ${ }^{6,17}$

\section{Celiac disease}

It could be amazing to know that the feces of untreated $\mathrm{CD}$ patients contain reduced concentration of Bifidobacterium species when compared with healthy individuals. Bifido bacterium infants has proven anti-inflammatory effects on $\mathrm{CD}$ intestine, it also can modify the Gliadin peptide sequence in vitro which could modify the pro inflammatory cascade triggered by Gliadin. One product named (USL\#3) contain 8 different bacteria has shown to decrease toxicity of gluten. It may help patients who are not adherent to a gluten free diet for life, in one controlled study showed improvement in symptoms and serum antibody levels. ${ }^{15}$

\section{Obesity \& Dyslipidemia}

Obesity incidence more than doubled between 1980-2014. Obesity is a chronic systemic inflammatory state that affects every cell in our body not just weight gain and imbalanced energy metabolism. Probiotics can break down bile salts in the gut and inhibit cholesterol reabsorption with $4 \%$ decrease in total cholesterol and 55\%decrease in $\mathrm{LD}$, after 8weeks.

Probiotics can affect body weight, improve insulin sensitivity and reduce chronic systemic inflammation. Akkermansia muciniphila has proved novel mechanism of action governing beneficial effects for obesity. ${ }^{18,19}$

\section{Central nervous system}

Microbiota-Gut-Brain axis had become a topic of great interest over the past years, the presumed communication between gastrointestinal and central nervous systems is still controversial. About 15 human randomized controlled trials found that certain commercially available strains of probiotics from the Bifidobacterium\& Lactobacillus genera when taken orally daily dose of 109 colony forming units (CFU) for 1-2 months resulted in good improvement in behavioral outcome and memory in some Neuro Psychological disorders (anxiety, depression, autism, and obsessive compulsive disorder). ${ }^{20}$

These findings are not systematic and univocal, but human studies are still limited and not yet conclusive. Further studies are needed to reach the suitable formulation, dosage and timing which can be considered the optimal regimen for each specific neurologic disorder and which age group can be targeted. Moreover, future trials should also stress on the tolerability and safety of use of various probiotics in those patients with neurologic diseases. ${ }^{21}$

\section{Conclusion}

- It seems that the probiotics dominate the human gut ecosystem has a huge impact on nutrients, micro nutrients, antigens, and inflammatory mediators.

- Many digestive and systemic diseases have an essential disorder in the intestinal epithelial function and microbiota balance.

- Despite the numerous in vitro and in mice benefits of probiotics as prophylaxis and treatment of many disease, researches on humans still needed.

- Probiotics seems to be a very promising and almost side effect free biologic medication when used in suitable dose.

- Effect of each probiotic seems to be strain specific. 
- The potential usefulness of probiotics in preventing or treating neurologic diseases still needs deeper investigation to help clarify the Aetiology and pathogenesis of ASD and other neurologic diseases which showed some benefit with prebiotics use. Welldesigned, randomized, controlled clinical trials are needed to confirm experimental results and to identify the appropriate strain, dose and timing for probiotic intervention. Then, a deeper evaluation of safety and tolerability should be carried out in additional studies.

\section{Acknowledgments}

None.

\section{Conflicts of interest}

Author declares there are no conflicts of interest.

\section{Funding}

None.

\section{References}

1. Mackowiak PA. Recycling Metchnikoff: Probiotics, the Intestinal Microbiome and the Quest for Long Life. Front Public Health 2013;1:52.

2. Araya M, Morelli L, Reid G, et al. Report of a Joint FAO/WHO working group on Drafting Guidelines for the Evaluation of Probiotics in Food, London, Ontario, Canada. April 30 and May 1. 2002.

3. Rijkers GT, de VOS WM, Brumner J, et al. Health benefits and health claims of probiotics: bridging scince and Marketing. Br J Nutr. 2011;106(9):1291-1296.

4. Allen SJ, Martinez EG, Gregorio GV, et al. Probiotics for treating acute infectious diarrhea. Cochrane Database Syst Rev. 2010;10(11): CD003048.

5. Nagler C. Gut bacteria that protect against food allergy identified. 2017

6. Michail S. The role of Probiotics in allergic diseases. Allergy, Asthma \& Clinical Immunology. 2009

7. Eloe-Fadrosh EA, Brady A, Crabree J, et al. Functional Dynamics of the Gut Microbiome in Elderly People during Probiotic Consumption. mBio . 2014;6(2):e00231-e00235.
8. Fuller R. Probiotics. The Scientific Basis. Chapman \& Hall. London. 1992.

9. Arvola T, Laiho K, Torkkeli S, et al. Prophylactic Lactobacillus GG reduces antibiotic-associated diarrhea in children with respiratory infections: a randomized study. Pediatrics. 1999; 104(5):e64.

10. Sarasawati S, Sitaraman R. Aging and the human gut microbiota-from correlation to causality. Front Microbiol . 2015;5: 764.

11. Wu GD, Chen J, Hofffmann C, et al. Linking long-term dietary patterns with gut microbial enterotypes. Science. 2011;334(6052):105-108.

12. Aragon G, Graham DB, Borum M, et al. Probiotic Therapy for Irritable Bowel Syndrome. Gastroenterol Hepatol (N Y) . 2010;6(1): 39-44.

13. Sung V, Hiscock, Tang MLK, et al. Treating infant colic with the probiotic Lactobacillus Reuteri: double blind, placebo controlled randomized trial. BMJ. 2014;20348:g 2107.

14. Alfaleh K, Anabrees J. Probiotics for prevention of necrotizing enterocolitis in preterm infants. Cochrane Database Syst Rev. 2011;10(4): CD005496.

15. Tavakkoli A, Green PH. Probiotic Therapy for Celiac Disease. J Clin Gastroenterol. 2013;47(2):101-103.

16. Kevin Whelan, Eamonn M. Probiotics in the Management of Irritable Bowel Syndrome and Inflammatory Bowel Disease. Curr Opin Gstroenterol . 2013;29(2):184-189.

17. Johnson K. Probiotic ‘Promising to’ Prevent and Treat Atopic Dermatitis. Medscape. 2017.

18. Agerholm-Larsen L, Bell ML, Grunwald GK, et al. The effect of a probiotic milk product on plasma cholesterol: a meta-analysis of shortterm intervention studies. Eur J Clin Nutr. 2000; 54(11): 856-860.

19. Kobyliak N. Conte C, Cammarota G, et al. Probiotics in prevention and treatment of obesity: a critical view. Nutrition \& Metabolism. $2016 ; 13: 14$

20. Wang H, Lee IS, Braun C, et al. Effect of Probiotics on Central Nervous System Functions in Animals and Humans: A Systematic Review. $J$ Neurogastroenterol Motl . 2016;22(4):589-605.

21. Umbrello G, Esposito S. Microbiota and neurologic diseases: potential effects of probiotics. J Transl Med. 2016;14:298. 